\title{
Schwannoma retroperitoneal benigno. Diagnostico incidental en paciente con hematuria del percusionista
}

\author{
I. Gómez García, R. Rodríguez Patrón, S. Conde Someso, E. Sanz Mayayo, C. Quicios Dorado, \\ *A. Palmeiro
}

Servicio de Urología, *Servicio de Anatomía Patológica. Hospital Ramón y Cajal. Madrid.

Actas Urol Esp 2005; 29 (5): 511-515

\section{RESUME N}

\section{SCHWANNOMA RETROPERITONEAL BENIGNO, DIAGNOSTICO INCIDENTAL EN PACIENTE CON HEMATURIA DEL PERCUSIONISTA.}

La incidencia de neoplasias retroperitoneales primitivos varía del 0,3 al 3\%. Los sarcomas suponen el grupo mas frecuente de neoplasias retroperitoneales, siendo el Schwannoma un tumor inusual con una incidencia del 1\% al 5\% del los tumores retroperitoneales primarios. El schwannoma también denominado neurinoma o neurolenoma, es un tumor derivada de las células de Schwann de los nervios periféricos. Se caracteriza por su inespecificidad clínica y radiológica, siendo el diagnostico patológico, con intensa positividad inmunohistoquímico a la proteína S-100. El tratamiento de elección es la exéresis quirúrgica, con márgenes amplios; no estando descrito casos de malignización ni de metástasis a distancia, pero si la existencia de recurrencia a nivel local probablemente secundaria a resección incompleta.

Palabras Clave: Schwannoma retroperitoneal, Laparoscopia, Sindrome de Von Ricklehausen.

\section{ABSTRACT \\ BENIGN RETROPERITONEAL SCHWANNOMA, I DIAGNOSE INCIDENTAL IN PATIENT WITH HEMATURIA OF THE PERCUSIONIST}

The incidence of retroperitoneal primitive tumour varies from the 0,3 to $3 \%$. The sarcomas suppose the group but it frequents of retroperitoneal tumour, being the Schwannoma an unusual tumour with an incidence from $1 \%$ to $5 \%$ of the retroperitoneal primary tumours. The schwannoma also denominated neurinoma or neurolenoma, it is a derived tumour of the cells of Schwann of the outlying nerves. It is characterized by their clinical and radiological inespecify, being the diagnose pathological, with intense positive inmunohistoquimics to the protein S-100. The election treatment is the surgical remove, with wide margins; not being described cases of malignización neither of metastasis at distance, but if the recurrence existence at probably secondary local level to incomplete resection.

Keywords: Retroperitoneal Schwannoma, Laparoscopy, Syndrome of Von Ricklehausen.

Los Scwannomas son tumores benignos derivados de las células de Schwann de la vaina de los nervios periféricos. Pueden originarse de cualquier nervio periférico, a excepción de los pares craneales I y II; la localización más frecuente es la cráneo facial, seguido de las extremidades, siendo la localización retroperitoneal excepcional.

\section{CASO CLINICO}

Varón de 26 años de edad, natural de Senegal, músico de profesión, sin antecedentes médicos, ni urológicos de interés, acude al servicio de urgencia por hematuria oligoasintomática de 3 años de evolución. No ha presentado episodios de dolor lumbar, ni de infección urinaria, no traumatismos lumbares, no sintomatología irritativa 
ni emisión litiásica; el paciente refiere que la hematuria se acentúa tras tocar "el tambor". Exploración física normal. Pruebas complementarias: hemograma, hemostasia normales; perfil bioquímico: elevación de la CPK resto normal; sistemático de orina, mioglobinuria; serología a diversos parásitos normal. Pruebas de imagen: ecografỉa urológica: riñón izquierdo normal, vejiga normal, riñón derecho: lesión dependiente del polo inferior de características mixtas que no afecta a la vía urinaria. UIV: normal. TAC: lesión dependiente del polo inferior del riñón derecho de contenido quístico (Fig. 1 y 2).

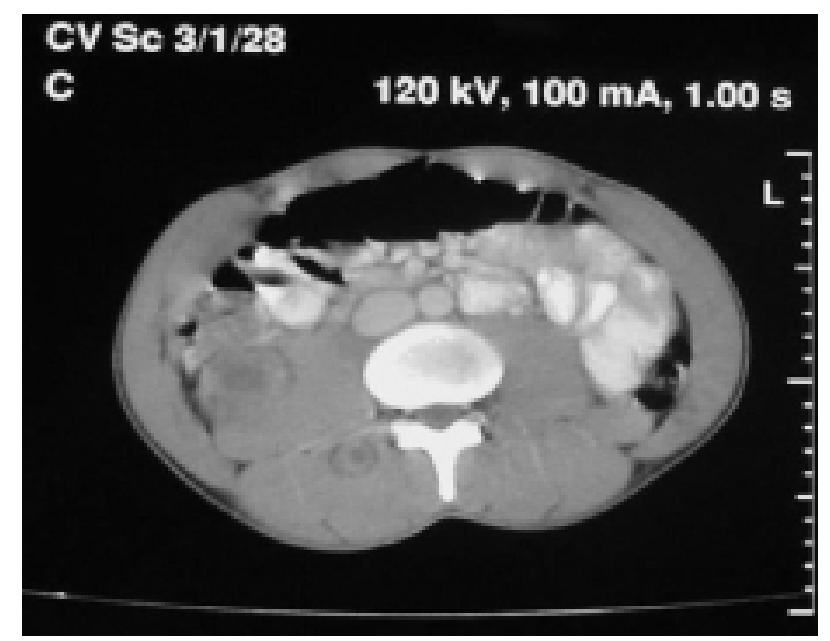

FIGURA 1: TAC lesión de aspecto quistico y bien delimitada sobre músculo psoas. Se aprecia otra lesión de iguales caracteristicas en musculatura paravertebral.
Con el diagnóstico de masa renal polar inferior derecha se desecha la posibilidad de cirugía laparoscópica, por la dependencia del riñón procediendo a lumbotomía subcostal, apreciando tumor bien delimitado, de consistencia pétrea, independiente del riñón, y dependiente del músculo psoas, siendo resecado con márgenes quirúrgicos amplios (Fig. 3).

El diagnóstico patológico fue de Schwannoma benigno, con intensa positividad a S-100, y objetivización de áreas A y B de Antoni (Fig. 4).

Seis meses tras la cirugía el paciente se encuentra asintomático y sin recidiva local.

\section{DISCUSION}

El Schawonnoma (SW) también denominado neurinoma o neurilemoma, es un tumor benigno, que se origina de las células de Schwann, de la vaina de los nervios periféricos. Verocay en 1910 hace la primera descripción de un tumor originado de nervio periférico, siendo Masson en 1932 quien acuñó la denominación de Schwannoma. Los SW, pueden aparecer en cualquier órgano o nervio del cuerpo, a excepción de los pares craneales I y II (olfativo y óptico), que carecen de células de Schwann. La localización más frecuente es la cráneo facial 40 al 50\%, seguido de las extremidades inferiores (30-35\%), siendo la localización retroperitoneal infrecuente $(0,5 \%$ y el $10 \%)$.

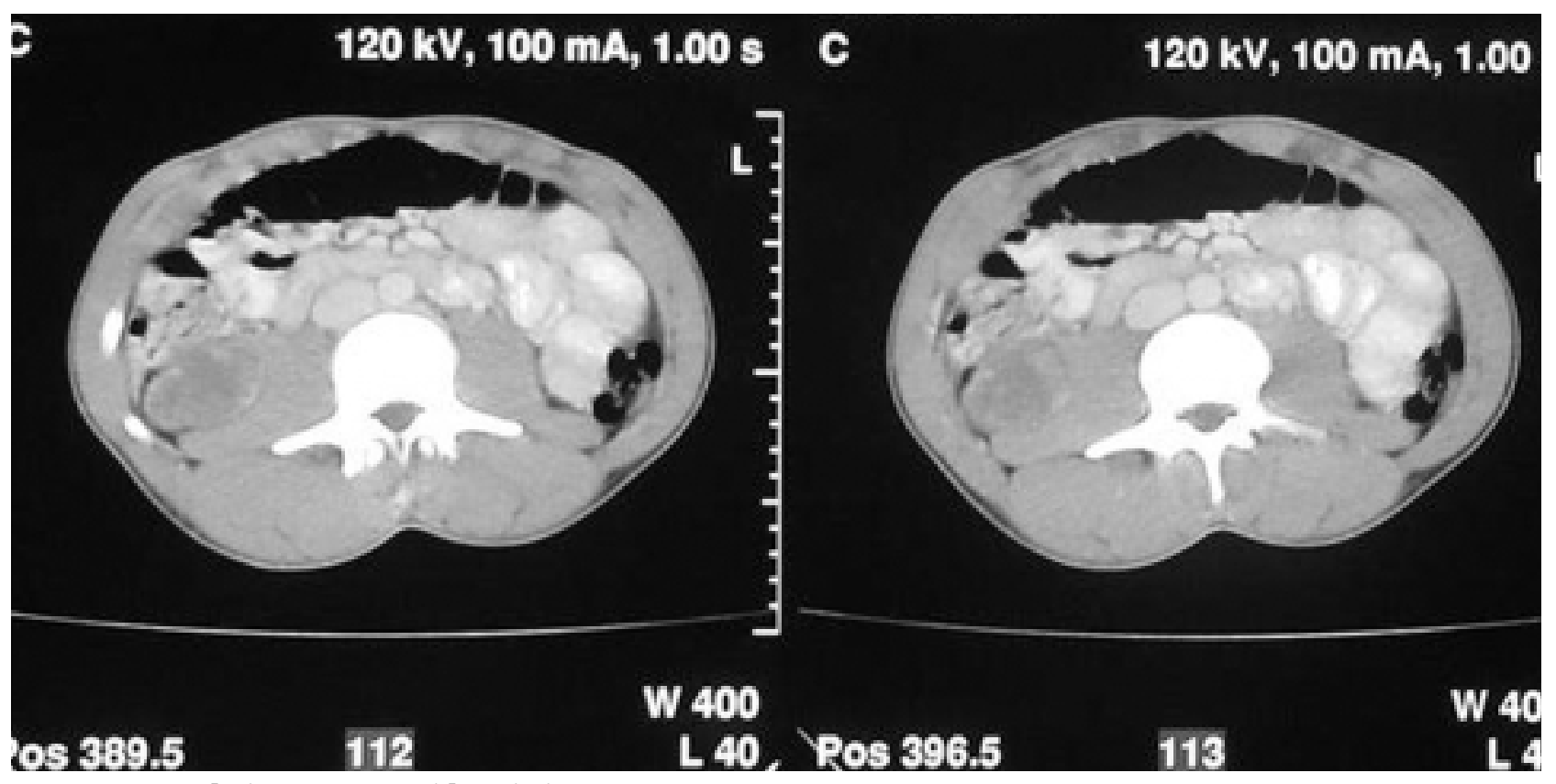

FIGURA 2: TAC lesión con contenido quistito. 


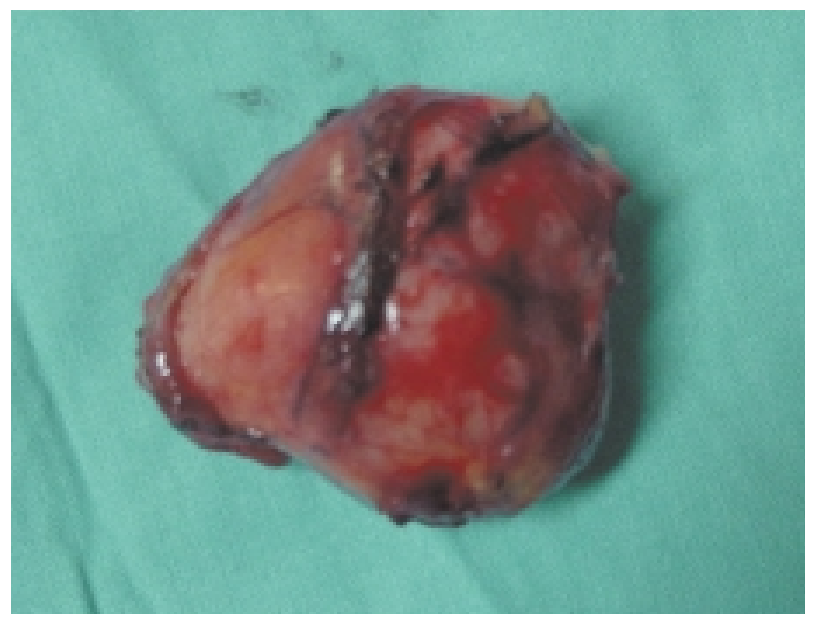

FIGURA 3: Imagen macroscópica del tumor.

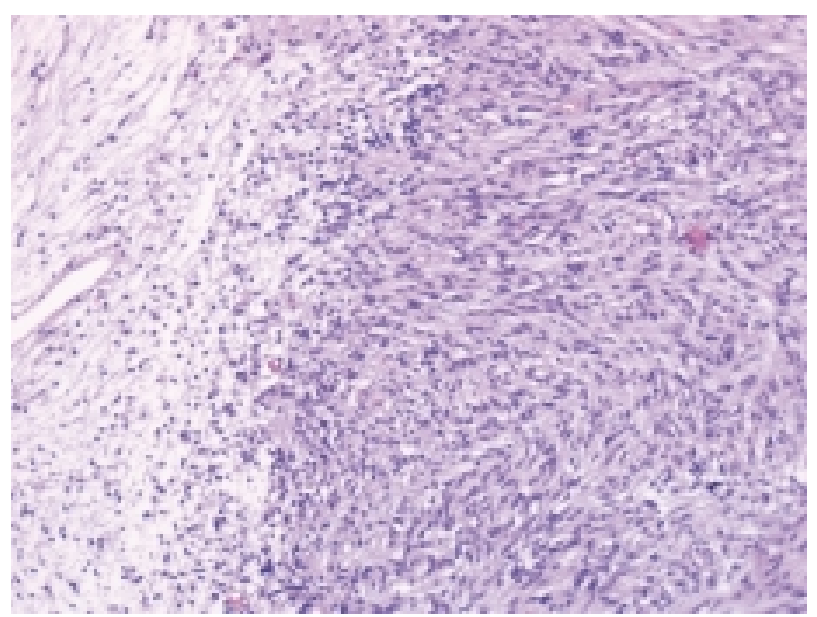

FIGURA 4: Imagen de microscopia donde se aprecian zonas $A$ con cuerpos de Verocay, con núcleos en empalizada; $y$ las zonas B con células mas separadas y escasas mitosis.

Los SW de localización retroperitoneal, se encuentran en la mayoría de los casos cercanos a la glándula adrenal, y por debajo de la arteria renal ${ }^{1-6}$.

La edad de aparición es diversa, estando casos descritos desde la segunda, hasta la séptima década de la vida, no existiendo diferencias entre $\operatorname{los} \operatorname{sexos}^{7,8}$. Los tumores retroperitoneales primarios son raros, con una incidencia que oscila entre $0,3 \%$ y el $3 \%^{5}$, suponiendo los SW entre el 1 y $5 \%$ de los tumores retroperitoneales ${ }^{4}$. En el sistema urogenital se han descrito casos en riñón, pene, cordón espermático, testículo, y túnica vaginal ${ }^{2,6,7}$. Esta descrito la asociación del SW, de un $5 \%$ a un $18 \%$, con la enfermedad de Von Recklinhausen, en cuyo caso suelen ser de localización atípica y de naturaleza maligna ${ }^{2,9}$.

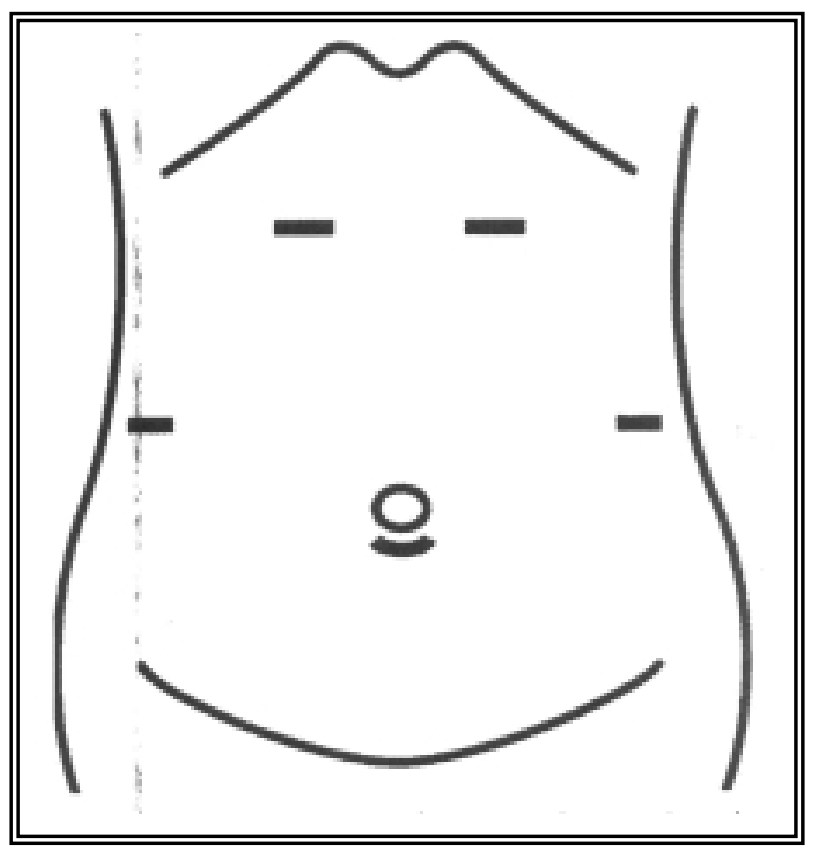

FIGURA 5: Disposición de puertos laparoscópicos para abordaje de tumor retroperitoneal, según Funamizu.

Clínicamente se caracterizan por su inespecifidad, siendo en la mayoría de las ocasiones asintomáticos, diagnosticándose de manera inciden$\operatorname{tal}^{5,7,8}$. De presentar sintomatología las mas frecuentes son las secundarias a la compresión de órganos y estructuras adyacentes, (uropatía, estreñimiento) ${ }^{2,4,7,9,11}$; existiendo casos descritos en los que el SW se extendía por medio de los espacios intervertebrales presentando dolor neurálgico como único síntoma ${ }^{3}$.

El diagnóstico preoperatorio es difícil, no existiendo métodos diagnósticos que aporten datos para un correcto diagnostico, teniendo que recurrir en muchos casos a la cirugía para el diagnóstico definitivo. El TAC y la ecografía, son útiles para determinar el tamaño, localización y el compromiso de estructuras vecinos, no existiendo imágenes específicas para el diagnóstico ${ }^{4,12}$. Los SW suelen tener imágenes quísticas, y presentar cambios degenerativos como, calcificaciones, signos de sangrado, y cambios hiali$\operatorname{nos}^{3,11,13}$. Otros autores que consideran la existencia de cambios quísticos en el seno de un tumor retroperitoneal es un hallazgo habitual en el SW 9; otros grupos consideran que la existencia de una masa heterogénea, con infiltración de órganos adyacentes, sugieren de tumor retroperi- 
toneal maligno. La realización de TAC con punción aspiración, no suele dar diagnostico preoperatorio concluyentes ${ }^{3,4,11,12}$. La punción guiada por TAC, es desaconsejada por la mayoría de los autores, debido a que son tumores con pleomorfismo celular, áreas de hemorragia, celularidad inflamatorio; que dependiendo del área de punción puede orientarnos hacia un incorrecto diagnóstico ${ }^{3,12}$.

Macroscópicamente son lesiones bien delimitadas, encapsuladas, de contenido heterogéneo, quístico, y de tamaño variable ${ }^{2,3}$. Histológicamente se aprecian dos tipos distintos de áreas. Las áreas tipo "A" o de Antoni se caracterizan por la existencia de células fusiformes que tienden agruparse en fascículos formando los cuerpos de Verocay; las áreas tipo "B", son zonas laxas de características mixoides, edematosa en las que existe poca o nula celularidad, formando en ocasiones áreas quísticas ${ }^{12}$. La positividad inmunohistoquímico a la proteína S-100 y la vimentina, es muy útil para su diagnóstico ${ }^{2,4,9,10}$; la determinación de la proteína $\mathrm{S}-100$ es utilizada para el diagnostico diferencial de los tumores derivados de la cresta neural ¿con los sarcomas? La determinación de S-100 es indispensable para el diagnostico definitivo y su diferenciación con los sarcomas y otros tumores mesenquimales.

El diagnostico diferencial debe realizarse con, los sarcomas y el SW celular, este ultimo definido histológicamente por la abundancia de áreas A sin existir las zonas tipo $\mathrm{B}^{10}$.

El tratamiento de elección es la cirugía, con resección completa de la masa y márgenes libres 1,3,7,9,10,14. En el momento actual el abordaje laparoscópico de estos tumores es una realidad, recomendando la utilización de un trocar Hasson infraumbilical de $10 \mathrm{~mm}$ y 4 puertos accesorios (Fig.5) ${ }^{4}$, otros autores defienden el acceso por retroperitoneoscopia utilizando tres trocares: un Hasson de $12 \mathrm{~mm}$ para la cámara, y dos trocares de trabajo de $5 \mathrm{~mm}^{5}$; siendo la limitación fundamental para la cirugía laparoscópicas es el tamaño tumoral, y no la localización anatómica del tumor ${ }^{4,5}$. La quimioteroterapia y radioterapia, no se han demostrado eficaces en el tratamiento de este tipo de neoplasias ${ }^{14}$.

No se han encontrado casos descritos en la literatura de metástasis a distancia ni de degene- ración; pero sí se describen pacientes con recidiva local después de la resección ${ }^{10}$, explicando las recurrencias por la persistencia de restos tumorales en el lecho de resección previa, pudiendo incluso recidivar cuando la resección es incompleta hasta en el $36,5 \%$ de los $\operatorname{casos}^{2,3,9,10}$. Es importante la vigilancia estricta de los pacientes con enfermedad de Von Recklihausen, ya que suelen asociarse a $\mathrm{SW}$ múltiples de localización excepcional, y con tendencia a la malignización.

\section{CONCLUSIONES}

Los SW retroperitoneales benignos son un tumor infrecuente no suponiendo mas del 5\% de los tumores retroperitoneales. Clínicamente se caracterizan por su inespecifidad, siendo la mayor parte de los síntomas secundarios a compresión de órganos o estructuras vecinas. Las técnicas de imagen solo aportan datos como localización, tamaño, y compromiso de estructuras vecinas, no existiendo datos radiológicos específicos en este tipo de tumores. Dado el pleomorfismo de estos tumores, la ocasional presencia de necrosis, áreas quísticas y zonas hemorrágicas, el uso de la PAAF no aportar datos concluyentes al diagnóstico. El diagnóstico es patológico mediante la observación del área A y B de Antoni y la determinación inmunohistoquímica positiva a la proteína S-100. En la actualidad el tratamiento de elección, es la resección tumoral con márgenes libres mediante cirugía laparoscópica. No existen casos descritos de metástasis ni de Malignizacion, pero si de recidiva local, en la mayor parte de los casos secundaria a una resección incompleta.

\section{REFERENCIAS}

1. Ziparo V, Bocchetti T, Mercantini P, Di Giacomo G, Caleno MP, Lucandri G. Retroperitoneal schwannoma: report of a clinical case and review of the literature G Chir. 1996;17(89):399-404

2. De Diego Rodríguez E, Roca Edreida A, Martín García B, Hernández Rodríguez R, Portillo Martín JA, Gutiérrez Baños JL. Schwannoma benigno retroperitoneal. Aportación de un nuevo caso. Actas Urol Esp 2000;24(8):685-688.

3. Daneshmand S, Youssefzadeh D, Chamie K, Boswell W, Wu N, Stein JP, Boyd S, Skinner DG. Benign retroperitoneal schwannoma A case series and review of the literature. Urology 2003;62:993-997.

4. Funamizu N, Sasaki A, Matsumoto T, Inomata M, Shiraishi N, Kitano S. Laparoscopic resection of a retroperitoneal Schwannoma behind the lesser omental sac. Surg Laparoscopic Endosc Percutan Tech 2004;14:175-177. 
5. Behrend M, Kaaden S, Von Wasielewski R, Frericks B. Benign retroperitoneal Scwannoma mimicking an adrenal mass. Surg Laparosc Endosc Percutan Tech 2003;13:133138.

6. Barrero Candau R., Ramírez Mendoza A., Morales López A., et al: Schwannoma benigno suprarrenal. Arch Esp Urol 2002; $7: 858-860$.

7. Cortes Aranguez I, Fernández Arjona M, De Castro Barbosa F, et al: Schwannoma retroperitoneal benigno: Revisión de la literatura y aportación de un nuevo caso. Actas Urol Esp 1999;23:621-624.

8. Pozo Mengual B, Perez Bustamante I, Fernández Fernández E, Mialdea Fernández R, Briones Mardones G, García-Cosio Piqueras M, Escudero Barrilero A. Schwannoma Retroperitoneal Benigno en paciente con antecedentes de tumor de Wilms en la infancia. Actas Urol Esp 2003; 27:379-382.

9. Micali S., Virgili G., Vespasiani G, et al: Benign schwannoma surrounding and obstructing the ureteropelvic junction. Eur Urol 1997;32:121-123.

10. Ortiz Rey J.A., Da Silva A., Rico Gala S.,et col: Schannoma celular retroperitoneal. Actas Urol Esp 1999;23:455-458.
11. Conde Sánchez JM, Espinosa Olmedo J, Camacho Martínez, Salazar Murillo R, Blasco Hernández P, Amaya Gutiérrez J, García Pérez M. Schwannoma presacro benigno que provoca uropatía obstructiva. Caso clínico y revisión de la literatura. Actas Urol Esp 2001;25:237-240.

12. Enzinger FN, Weiss SW: Malignant tumours of the peripheral nerves, in Enzinger FN, and Weis SW (Eds): Soft tissue tumours, 3rd ed. St. Louis, CV, Mosby 1995;821-832.

13. Takatera H, Takiuchi H, Namiki M, Takaha M, Ohnishi S, Sonoda T: Retroperitoneal Schwannoma. Urology 1986; 28: 529-531.

14. Ishii N, Sawamura Y, Tada M, Acoustic cellular schwannoma invading the petrous bone: case report. Neurosurgery. 1996;38:576-578.

Dr. I. Gómez García

María Tubau, 15

28050 Madrid

(Trabajo recibido el 3 de marzo de 2005) 